\title{
Espace public, identité et nation au Québec : mythes et méprises du discours souverainiste
}

\section{Daniel Salée}

Numéro 25, 1995

Être ou ne pas être québécois

URI : https://id.erudit.org/iderudit/1002294ar

DOI : https://doi.org/10.7202/1002294ar

Aller au sommaire du numéro

\section{Éditeur(s)}

Département de sociologie - Université du Québec à Montréal

ISSN

0831-1048 (imprimé)

1923-5771 (numérique)

Découvrir la revue

Citer cet article

Salée, D. (1995). Espace public, identité et nation au Québec : mythes et méprises du discours souverainiste. Cahiers de recherche sociologique, (25), 125-153. https://doi.org/10.7202/1002294ar
Résumé de l'article

Ce texte propose une lecture critique du discours souverainiste sur la recomposition de l'espace public national québécois. L'auteur soutient essentiellement que la redéfinition de la nation souhaitée par les souverainistes québécois selon laquelle le Québec doit tendre vers l'accomplissement d'une nation dé-ethnicisée, fondée sur une logique civique et rationnelle, est en porte-à-faux par rapport à l'imaginaire social des Québécois et la réalité politique du Québec. Sous couvert d'adhésion à la raison universelle, cette redéfinition repose sur une mise au ban de l'ethnicité et des particularismes culturels et participe d'une volonté de subsomption à la fois de l'identité historique des Québécois et des identités autres qui composent désormais le tissu social du Québec en un espace public post-national et homogénéisant. Contrairement aux apparences, le sens de la nation qui dérive du projet théorico-politique des souverainistes cache en réalité un acte d'exclusion et des prétentions hégémoniques au profit de la nation historique. L'auteur soutient qu'il ne sert à rien d'évacuer la différence et l'altérité inhérentes à la dynamique politique québécoise. L'ethnicité et la culture sont des faits de conscience incontournables. Un véritable projet d'avant-garde de recomposition de l'espace public au Québec devrait plutôt chercher à en accepter les aboutissants et à réaliser une configuration institutionnelle flexible qui ne banalise pas l'altérité et la différence, qui ne hiérarchise pas les identités et qui ne soit pas fondée sur une conception préétablie de l'espace public. Cela implique l'abandon de la " Nation " comme pivot axiomatique de la communauté politique et une remise en question fondamentale des paramètres du libéralisme contemporain.
Ce document est protégé par la loi sur le droit d'auteur. L'utilisation des services d'Érudit (y compris la reproduction) est assujettie à sa politique d'utilisation que vous pouvez consulter en ligne.

https://apropos.erudit.org/fr/usagers/politique-dutilisation/ 


\title{
Espace public, identité et nation au Québec: mythes et méprises du discours souverainiste
}

Daniel SALÉE

\begin{abstract}
[...] le Québec s'est défendu comme il a pu, usant de moyens subtils dont le meilleur fut de donner au monde et au reste du Canada de fausses images de lui-même. Ce moyen avait des inconvénients, en particulier celui de nous mystifier nous-mêmes.
\end{abstract}

\section{JACQUES FERRON, Du fond de mon arrière-cuisine.}

La production sociographique et historiographique québécoise des quinze dernières années s'est, dans l'ensemble, constamment employée - parfois délibérément, parfois implicitement - à démontrer que le Québec a été de tout temps une société «normale», subissant les aléas de l'histoire selon des modalités semblables à celles qui existent dans toute autre société occidentale. Prenant le contre-pied de thèses et d'ouvrages qui n'avaient de cesse d'insister sur le caractère traditionaliste, pré- ou anti-moderne d'une société prétendument incapable de voir autrement que par la lorgnette d'une identité étroite, limitative et archaïquement conçue, le discours savant des dernières années a généralement cherché à infirmer les postulats d'une sociographie et d'une historiographie surannées pour s'engager dans une entreprise de revalorisation de l'image sociale et historique du Québec. Cette revalorisation ne sera pas nécessairement a-critique; rompant avec les modes lourdement nationalitaires et culturalistes d'appréhension de la réalité socio-historique du Québec qui avaient dominé le champ discursif jusqu'alors ${ }^{1}$, elle sera surtout tournée vers la démonstration du caractère éminemment moderne, ouvert, pluraliste, hétérogène et socialement complexe de la société québécoise ${ }^{2}$.

1 Voir D. Salée, «L'analyse socio-politique de la société québécoise: bilan et perspectives», dans G. Boismenu et autres, Espace régional et nation. Pour un nouveau débat sur le Québec, Montréal, Boréal Express, p. 15-49.

2 Jocelyn Létourneau a noté récemment à propos de la production historienne: «En fait, la recherche des quinze dernières années a fait littéralement éclater, simultanément, le vieux récit canadien-anglais (fortement articulé au discours de l'élite bourgeoise et 
Le Québec comme société normale donc: disparues du discours les topiques d'une certaine différence socioculturelle et d'une identité ethnolinguistique en mal de reconnaissance politique forçant à un positionnement justificatif constant. Il ne s'agit plus désormais d'affirmer la nation dans sa subjectivité historiquement déterminée et singulière: du fait de son accession à la modernité, le Québec existe comme communauté nationale politique ${ }^{3}$ qui n'a plus à légitimer sa spécificité ni à faire valoir les paramètres de son existence à la face du monde. Le Québec est tout simplement.

Certes, le passage d'une vision idiosyncratique du Québec à une vision plus globale et universelle n'est pas sans sous-entendus politiques: si le Québec existe comme une société normale, comparable à toute autre, cela ne justifie-t-il pas ses aspirations souverainistes, sa volonté d'autodétermination? Le sous-texte nationalitaire qui animait l'historiographie et la sociographie d'avant 1980 n'est jamais très loin, quelle que soit la manière dont on reformule l'analyse du Québec. Normal, diront certains, tant que le Québec n'aura pas acquis sa pleine indépendance politique, il ne saurait en être autrement. Quoi qu'il en soit, il est un aspect remarquable de la reformulation des dernières années: la question nationale - au sens traditionnel de l'affirmation historique de la subjectivité identitaire québécoise - ne se situe plus nécessairement au centre du regard sociologique que l'on porte aujourd'hui sur le Québec ${ }^{4}$. Sans avoir été complètement éliminée du champ discursif, elle semble plus souvent subsumée dans une série de

nationaliste anglophone) et le vieux récit canadien-français (intimement lié au discours nationaliste des élites francophones, et ce, depuis toujours) [...] l'apparence générale du Sujet québécois a été "normalisée", son état existentiel et son parcours historique s'apparentant désormais à celui des Autres tout en ne s'y réduisant pas, spécificité oblige... Désormais prévalent les idées de similitude, de convergence et de parité: la frontière du Nous-Autres, et donc l'identitaire québécois, sont en voie d'être établis sur d'autres bases.» (J. Létourneau, «La production historienne courante portant sur le Québec et ses rapports avec la construction des figures identitaires d'une communauté communicationnelle», Recherches sociographiques, vol. 36, no 1, 1995, p. 18, 20-21. Voir aussi R. Rudin, «La quête d'une société normale. Critique de la réinterprétation de l'histoire du Québec», Bulletin d'histoire politique, vol. 3, no 2, 1995, p. 9-42.)

3 G. Bourque, «Société traditionnelle, société politique et sociologie québécoise, 1945-1980», Cahiers de recherche sociologique, no 20, 1993, p. 49.

4 La publication depuis trois ou quatre ans de recueils et d'ouvrages de synthèse portant sur les aspects les plus divers de la réalité sociale québécoise en témoigne. Voir en particulier G. Daigle (dir.), avec la collaboration de G. Rocher, Le Québec en jeu. Comprendre les grands défis, Montréal, Presses de l'Université de Montréal, 1992; F. Dumont, S. Langlois et Y. Martin (dir.), Traité des problèmes sociaux, Québec, IQRC, 1994; A.-G. Gagnon (dir.), Québec: État et société, Montréal, Québec/Amérique, 1994; G. Lachapelle et autres, The Quebec Democracy: Structures, Processes and Policies, Toronto, McGraw-Hill Ryerson, 1993. 
réflexions implicites et d'analyses plus larges sur le sens de la citoyenneté, le renouvellement de la démocratie, la tension entre droits individuels et droits collectifs et l'insertion du Québec dans la tendance irrépressible à la mondialisation, bref sur la nature de l'espace public dont doit se doter le Québec.

Cette mise à jour de la parole sociographique n'a pas été sans influence sur la parole politique elle-même. Au cours des dernières années, le sens théorique qui aiguillonne le nationalisme des souverainistes québécois s'est considérablement transformé. Bien qu'il continue d'interpeller les francophones, il se veut maintenant beaucoup plus large, beaucoup plus englobant et authentiquement préoccupé par les problèmes urgents que pose la pluriethnicité à la société québécoise. Le nationalisme officiel est aujourd'hui délesté des connotations ethnoculturelles qui, il y a une décennie à peine, l'associaient exclusivement à la majorité française du Québec. Il s'agit pour les souverainistes d'édifier un État souverain; un État francophone certes, mais surtout un État démocratique auquel devraient pouvoir se rallier tous les membres de la société sans exception et sans exclusion aucune. La nation ne doit plus s'entendre dans son sens restreint et culturellement déterminé, mais au sens de la caution légale et territoriale que seul un État dûment constitué peut lui prêter.

En principe, il y a tout lieu de se réjouir du caractère humaniste et en apparence généreux du projet souverainiste. Qui oserait être contre la vertu, contre un horizon moral aussi vaste et obligeant? Néanmoins, une certaine perplexité est de mise. Non pas tant à l'égard de l'idée de la souveraineté du Québec, mais plutôt quant à la manière dont elle est présentée par les élites politiques et justifiée par l'imagination sociologique. L'espèce d'empressement que met aujourd'hui le discours public à évacuer la problématique identitaire et les apories de l'ethnicité - sur laquelle le Québec moderne s'est pourtant imaginé et construit - participe de cette tendance à «nous mystifier nous-mêmes» que notait jadis Jacques Ferron.

Le présent texte propose une lecture critique à la fois du souverainisme post-national qui est présentement offert à la population du Québec et des rationalisations qu'une certaine imagination sociologique invoque à son appui. Quatre postulats dirigent la démarche: 1) l'idée d'une nation québécoise «dé-ethnicisée», essentiellement civique et rationnelle, est en porte-à-faux par rapport à l'imaginaire social québécois; 2) elle repose sur une fiction idéologique à laquelle les élites politiques et intellectuelles souverainistes n'adhèrent même pas véritablement; 3 ) cette fiction s'inscrit dans une perspective limitée et contraignante de la démocratie et de la citoyenneté; 4) les responsables de la formulation du projet souverainiste risquent de 
répéter les errements de l'Histoire s'ils continuent à faire l'épargne d'une analyse en profondeur des paramètres réels de la société québécoise et d'une reconsidération des fondements théoriques sur lesquels ils entendent constituer l'espace public québécois de demain.

\section{Exit l'ethnicité}

Thuriféraire inlassable de la cause souverainiste, la politologue Anne Legaré expliquait dans un texte récent que le Québec en est pour ce qui touche son développement politico-institutionnel à ce point où le rapport entre l'État et la nation s'est modifié «en ouvrant cette dernière du côté de la rationalité au détriment de 1'“ethnicité5"». Le Québec aurait en quelque sorte accompli avec succès son passage de la «nationculture» à la «nation-raison». À la fois théorique et programmatique, son texte est caractéristique de l'orientation politique que les souverainistes semblent vouloir imprimer à l'espace public québécois et mérite donc que l'on s'y arrête.

La démarche de Legaré est ici motivée par une volonté clairement exprimée de répliquer aux détracteurs de la souveraineté qui y voient dérive nationaliste, aspiration ethnique appuyée exclusivement sur la langue et la culture et repli sur soi. Le nationalisme québécois et les aspirations souverainistes qu'il alimente ne seraient rien de tout cela. Au contraire, soutient Legaré, engagé dans une entreprise largement nationalitaire depuis les années soixante, le Québec a démontré, et ce en grande partie sous l'influence du mouvement nationaliste, qu'il était essentiellement pluraliste et ouvert aux autres cultures qui y cohabitent. Cela se vérifie empiriquement dans sa conception de la démocratie fondée sur les droits politiques et la non-discrimination culturelle ou ethnique, conception qui se concrétise par des lois et des dispositions administratives et institutionnelles précises à cet égard. Le mouvement nationaliste incarné par le Parti québécois et le Bloc québécois n'a jamais été guidé par autre chose qu'un respect exhaustif des droits des minorités vivant en sol québécois, la reconnaissance de leurs droits sociaux et culturels et la volonté de protéger leurs héritages spécifiques. «Entretenir le doute sur ces faits, avertit Legaré, c'est extrapoler de façon inappropriée les retombées des nationalismes ethniques qui font suite à la chute de l'empire soviétique ou, encore, c'est projeter sur la communauté francophone un regard de suspicion, inspiré de siècles de domination, à l'effet que cette collectivité serait incapable d'égalité6.»

5 A. Legaré, «La souveraineté: nation ou raison?», dans A.-G. Gagnon (dir.), ouvr. cité, p. 46.

6 Ibid., p. 42. 
Pour Legaré, la nation n'est pas a priori un donné culturel. Elle n'est pas marquée de façon indélébile au coin d'un vécu historique précis ou de composantes ethnolinguistiques particulières. «La nation est un processus en constante définition par son lien intime avec le social et le culturel» et au Québec, «elle est liée à la complexité culturelle changeante et différenciée du tissu social québécois». Ainsi, tous les éléments qui en constituent la trame (francophones, anglophones, immigrants, autochtones) «doivent s'associer de nouveau de façon consensuelle au cœur d'une redéfinition de la nation ${ }^{7}$ ».

Cette redéfinition de la nation, il va sans dire, passe par la constitution d'un État québécois souverain. L'État souverain forme en effet l' «horizon de rationalité de ce processus», le creuset à l'intérieur duquel la rencontre des divers éléments constitutifs de la société peut s'accomplir. Pour ce faire, la citoyenneté se pose comme le fondement de la construction, de la reconfiguration, de la nation; elle assure la cohésion sociale et «désigne le nouveau sujet collectif qui régit les droits et obligations inscrits dans la constitution»; elle «assure l'égalité de tous à l'endroit du bien commun»; de même, «sanctionnée par ceux qu'elle désigne, elle fonde la légitimité sur la reconnaissance mutuelle d'une égalité au-delà de la diversité des intérêts de chacun»; bref, la citoyenneté à la base de la souveraineté rend la nation politique possible. En clair,

[...] la redéfinition de la nation [...] implique la primauté de la citoyenneté sur toute représentation culturelle excluante de ce nouveau sujet politique. L'imaginaire politique qui préside à la volonté souverainiste est donc celui d'une nation ouverte et élective, basée sur l'égalité dans la citoyenneté [...]. La nation québécoise ne se suffit donc pas d'être «naturelle» [...]. Elle deviendra le véritable sujet politique de l'État québécois lorsque, par la souveraineté, elle réunira un consensus qui inclura les minorités nationales dans un nouveau pacte légitime autour du futur État souverain. [...] par conséquent [...] la conscience souverainiste [...] se traduit mieux par la notion de rationalité subjective que par l'idée de nationalisme. Par rationalité subjective, nous entendons l'intériorisation par les citoyens des éléments rationnels d'une transformation caractérisée par un projet d'auto-institution du politique. En d'autres mots, il ne s'agit pas de l'expression d'une identité culturelle préexistante mais de la prise en considération par le citoyen du Québec d'une rationalité qui commande des réaménagements majeurs du système politique ${ }^{8}$. 
Le parti pris rationaliste qu'affiche Legaré relève essentiellement de la volonté d'épurer le projet souverainiste et, par conséquent, la nation des scories identitaires qui risqueraient inévitablement de nuire au mouvement d'auto-institution de l'État québécois en l'engageant dans «une dérive passéiste, ethnocentrique ou romantique sur le modèle des nationalismes qui ont pris tristement la relève depuis l'éclatement de l'empire soviétique ${ }^{9}{ }^{\natural}$. Bien sûr, il s'agit aussi de convaincre les sceptiques que les souverainistes québécois ne ressemblent en rien à cette engeance antidémocratique, obsédée par la promotion exclusive et hégémonique de la francité au Québec et qui, selon les nombreux critiques du nationalisme québécois, dominait la construction de la nation. En prétendant que le projet souverainiste s'inscrit d'abord dans un imaginaire étatique, démocratique et universel, fondé sur la primauté du lien politico-juridique par rapport au lien culturel, Legaré nous assure que les souverainistes nouvelle manière récusent l'ethnicité tare indicible dont ils se défendent bien d'être affligés. Elle nous demande finalement de croire au projet souverainiste qui, puisqu'il est basé sur la raison (raison politique et raison du droit), offre nécessairement toutes les garanties contre les excès possibles de la nation ethnique.

\section{Avez-vous (bien) lu Renan?}

Le rejet des particularismes dont l'identité ethnique est une manifestation n'a rien de nouveau. Il s'inscrit en filigrane de la pensée politique occidentale depuis que le libéralisme s'est imposé comme mode dominant de conception de la société. À partir du début du XIXe siècle en particulier, les nations modernes se sont constituées dans et par la mise en place d'institutions étatiques vouées à l'unification et à l'homogénéisation des agents sociaux disparates dans les formations sociales pré-modernes. En fait, l'insertion dans la modernité a toujours largement dépendu de l'accomplissement d'un procès institué d'uniformisation socio-économique. En ce sens, les positions théoricopolitiques qu'expose Legaré s'inspirent directement des acquis de l'expérience occidentale. On ne se surprendra donc pas qu'elle ait puisé chez un auteur comme Ernest Renan les fondements théoriques de sa révision relativement au sens de la nation québécoise.

Le célèbre essai de cet historien français du XIXe siècle, $Q u$ 'est-ce qu'une nation? (1882), a été le point de départ d'un bon nombre de réflexions et d'analyses autour du phénomène nationalitaire depuis plusieurs décennies. L'histoire de la pensée a retenu le nom d'Ernest

$9 \quad$ Ibid., p. 50. 
Renan pour sa théorisation de la nation politique et sa dénonciation de la confusion intellectuelle entre nation et ethnie. La nation renanienne se propose comme une communauté idéale de citoyens égaux entre eux, une communauté qui ignore les particularités ethniques, régionales, culturelles, linguistiques et religieuses de chacun. Rien n'en distingue les membres si ce n'est une langue et une histoire communes ainsi qu'une volonté de vivre ensemble. Dans le débat sur la question du sens de la «nation française» (civique et républicaine) opposé à celui de la «nation allemande» (ethnique et romantique ${ }^{10}$ ), Renan est rangé d'emblée du côté de la première et donné comme l'incarnation théorique de l'idéal égalitaire, démocratique et post-jacobin de la nation moderne.

On comprend donc qu'aujourd'hui Renan soit volontiers évoqué par qui veut affirmer le caractère non ethnique de la nation moderne; l'auteur sert de caution morale et intellectuelle pour justifier tout en les atténuant les ambitions politiques du nationalisme. Puisque, suivant la perspective de l'historien, le désir de nation n'apparaît plus comme la célébration de particularismes identitaires étroits et exclusivistes, mais bien comme une forme achevée et désirable d'accomplissement démocratique, la volonté nationaliste s'impose comme un projet politique tout à fait respectable. En ces temps blafards et honteux de purification ethniques à l'Est, mieux vaut délester la nation d'une ethnicité qui peut devenir politiquement gênante, surtout à l'intérieur de cadres institutionnels qui se veulent démocratiques.

Pourtant, quoi qu'il en soit des efforts pour masquer les traces de l'ethnie, celles-ci semblent toujours réapparaître en arrière-fond sur le canevas de la nation. Dans sa conception même de la nation, Renan n'était pas plus que quiconque à l'abri des relents identitaires qui interpellent la conscience profonde de chaque individu. Il semble, par exemple, que l'historien ait été profondément influencé par l'historicisme romantique allemand - un important courant historiographique à son époque - et la compréhension germanique du phénomène nationalitaire ${ }^{11}$, celle-là même qu'on oppose aujourd'hui à la vision rationaliste de la nation moderne prétendument ancrée dans la perspective renanienne. En fait, une lecture attentive et plus analytique

10 Voir, sur cette question, D. Schnapper, «Ethnies et nations», Cahiers de recherche sociologique, no 20, 1993, p. 157-167, et La communauté des citoyens. Sur l'idée moderne de nation, Paris, Gallimard, 1994; M. Wieviorka, La démocratie à l'épreuve. Nationalisme, populisme, ethnicité, Paris, La Découverte, 1993.

11 M. Thom, «Tribes within nations: The ancient Germans and the history of ancient France», dans H. K. Bhabha (dir.), Nation and Narration, Londres, Routledge, 1990, p. 23-43. 
révèle l'existence d'une problématique éminemment ethnoculturelle au cœur même de la conceptualisation renanienne de la nation ${ }^{12}$.

Lorsque l'historien proclame qu' «une nation est une âme, un principe spirituel», on voit mal comment l'imaginaire étatique peut donner un sens à cette âme, à ce principe spirituel. Ne s'agirait-il pas plutôt de concevoir le processus à l'inverse? Dans la mesure où l'État participe de l'activité humaine, des interactions diverses entre agents sociaux, c'est l'État qui doit logiquement émerger du principe spirituel et donc de la nation. La nation doit avoir conscience d'elle-même avant de chercher à s'instituer en des mécanismes étatiques. Or d'où vient cette conscience de soi? La réponse de Renan est éloquente:

La nation, comme l'individu, est l'aboutissant d'un long passé d'efforts, de sacrifices et de dévouements. Le culte des ancêtres est de tous le plus légitime; les ancêtres nous ont faits ce que nous sommes. Un passé héroïque, des grands hommes, de la gloire (j'entends de la véritable), voilà le capital social sur lequel on assied une idée nationale ${ }^{13}$.

Difficile d'imaginer que Renan ait pu avoir en tête autre chose que la culture et l'ethnicité lorsqu'il a écrit ces mots. La nation, c'est d'abord une somme historique; la somme des bons et des mauvais coups qu'a eu à subir une communauté d'acteurs sociaux réunis dans un espace géographique donné; la somme des manières de faire face à l'adversité, des manières d'être dans les relations sociales. Bref, la nation est d'abord et avant tout une somme culturelle. Il est étonnant qu'on ait voulu faire dire à Renan autre chose que cela.

Dans son désir de délester définitivement la nation de ses oripeaux ethnoculturels et passéistes, Anne Legaré nous annonce que la nation n'est pas un donné culturel, qu'elle peut changer selon les aléas de la conjoncture ou de la recomposition du tissu social. Ce qui change, en fait, c'est la manière d'exprimer pour soi et pour les autres l'identité que recouvre la nation - au Québec, nous nous sommes d'abord dits canadiens, puis canadiens-français, puis enfin québécois. Les fondements de cette identité, c'est-à-dire les balises culturelles, linguistiques, ethniques et territoriales de la nation, ne se modifient que très peu. Ils restent pour ainsi dire invariables à travers le temps.

«No nation imagines itself coterminous with mankind», a écrit Benedict Anderson dans son admirable ouvrage Imagined Commu-

12 Voir M. Cahen, Ethnicité politique. Pour une lecture réaliste de l'identité, Paris, L'Harmattan, 1994, chap. VI.

13 E. Renan, «Qu'est-ce qu'une nation?», dans E. Renan, Euvres complètes, t. I, édition établie par H. Psichari, Paris, Calmann-Lévy, 1947, p. 904. 
nities $^{14}$. La nation se définit, s'imagine, inévitablement à l'exclusive selon des critères identitaires généralement clairs, historiquement déterminés, d'ethnicité, de langue et de territorialité, bref, de culture. Il n'est pas nécessairement impossible d'y pénétrer ou d'y adhérer, mais cela ne s'accomplit presque toujours qu'au prix de l'abandon, de la renonciation ou de la mise en veilleuse de son identité antérieure. Le «vouloir vivre ensemble» de Renan n'implique pas, dans le cadre strict de la nation, la transculturalité ni même l'interculturalité. Il est fondamentalement uniculturel, c'est-à-dire qu'il s'appuie sur des dénominateurs sociétaux communs, tissés par et à travers l'histoire, auxquels les membres du groupe national adhèrent d'emblée et par lesquels ils se reconnaissent une communauté d'être, communiquent et interagissent en relative harmonie. La volonté de vie commune ne s'improvise pas, elle ne se manufacture pas. Elle se manifeste surtout au sein de communautés dont les membres partagent la même expérience historique, la même culture, c'est-à-dire une manière de code social (code de comportement, de communication, voire code vestimentaire) ancré dans les tréfonds de la conscience individuelle et collective et que seule l'appartenance non équivoque à la communauté permet de comprendre et de maîtriser. Cela ne veut pas dire que le contenu de ce code soit figé et immuable. De nouveaux codes, de nouvelles expressions culturelles peuvent apparaître, nés de la diversité, voire du métissage d'une société, mais avant qu'un nouveau code en arrive à dominer, et donc à s'exprimer dans une volonté nouvelle de vie commune, il faudra que l'ancien code ait perdu toute pertinence. Cela suppose un processus social de longue durée, tissé à même la réalité des rapports sociaux et des rapports de pouvoir, et ancré dans la renégociation constante de l'espace public commun - rien, on l'aura compris, que la création de toutes pièces d'un État nouveau ne puisse régler instantanément.

Dans leur souci politique de présenter une image qui corresponde aux canons d'un libéralisme bon teint qui postule une adéquation irréfléchie entre ferveur ethnoculturelle et antidémocratisme, les souverainistes québécois ont délaissé les fondements ethniques de la nation québécoise pour s'inventer un profil idéologique plus rentable politiquement. Mais le fait est que la nation émerge toujours du passé et que le désir de nation tient moins à un projet politique précis qu'aux systèmes culturels qui l'ont précédé et qui l'animent en définitive ${ }^{15}$. En d'autres mots, «il ne faut pas confondre projet politique et réalité: la nation-forme d'ethnicité correspond plus exactement à la réalité des gens, c'est-à-dire à la réalité des faits de conscience, quels que soient les

14 B. Anderson, Imagined Communities, Londres, Verso, 1991, p. 7.

15 Ibid., p. 12. 
projets politiques de tel ou tel secteur de l'élite ${ }^{16_{»}}$. En reparcourant le chemin intellectuel que semblait avoir suivi Renan jadis à propos de la nation, les souverainistes québécois suivent une fausse piste.

\section{Être ou ne pas être une nation?}

Il y a bien, à n'en pas douter, quelque chose de séduisant dans un projet de redéfinition de la nation qui pose la primauté de la citoyenneté et de la rationalité sur l'ethnicité et le particularisme culturel. La mise en place d'un État souverain dans lequel chacun, toutes origines et toutes allégeances identitaires confondues, se reconnaîtrait d'emblée faciliterait de beaucoup, en effet, les transactions socio-politiques. L'ethnicité, surtout lorsqu'elle s'affiche comme un mode privilégié d'expression et de revendication politique, n'est pas sans créer, il faut bien l'avouer, des barrières difficiles à franchir dans la construction d'une culture et d'un espace publics communs. Les choses seraient plus simples, est-on naturellement porté à croire, si ces différences qui distinguent mais qui tiennent aussi l'Autre à distance ne comptaient pas vraiment - éternelles apories de l'idéal libéral.

En dépit de son attrait objectif, le projet souverainiste dans sa facture théorique actuelle constitue un leurre. La chose n'est pas nécessairement délibérée, mais elle repose néanmoins sur une lecture inadéquate du réel politique et des paramètres actuels de la sociétalité québécoise. Affirmer le primat de la raison étatique, aussi noble, généreuse et égalitaire fût-elle, sur la raison culturelle, tout en continuant d'invoquer la nation, procède de manœuvres discursives mystifiantes. On joue ici sur plusieurs registres, mêlant confusément impulsion démocratique, idéaux égalitaires et promotion de la communauté imaginée d'origine. En bout de piste, la nation que l'on croit être n'est pas la nation que l'on dit vouloir.

Entre la raison politique que les souverainistes voudraient voir triompher et la réalité des rapports sociaux et de la dynamique politique actuelle, il y a un écart assez grand, et leur construit théorique, dans toute sa netteté et sa rigueur abstraite, n'en tient pas compte. Ce construit est presque trop parfait. Il suppose des acteurs sociaux une rationalité qui ne se vérifie pas dans la manière dont ils prennent position face aux enjeux socio-politiques qui traversent la société québécoise. Ce qui ne signifie pas que les acteurs sociaux ne sont pas rationnels. Simplement, la rationalité qui les guide est celle de leurs intérêts spécifiques, souvent étroits: celle d'un vécu immédiat, subjectif, irrémédiablement marqué au fer rouge d'une identité historiquement

16 M. Cahen, ouvr. cité, p. 11. 
chargée dont ils n'accepteraient pas de se départir. Or, si on s'en rapporte au texte de Legaré, la rationalité à laquelle les souverainistes font implicitement référence est celle de la raison transcendante, objective, comme s'il ne pouvait y avoir qu'une seule rationalité valable, qu'une seule logique acceptable parce que moralement supérieure. Pareille rationalité n'est bien souvent rien de plus qu'une fiction montée de toutes pièces par le pouvoir technocratique ${ }^{17}$. Elle est rarement opérante de toute façon au niveau de la société civile où elle est généralement contestée par de nombreux groupes qui en remettent en question l'impérialisme théorico-intellectuel.

Le fantôme de Renan plane manifestement sur cette vision des choses. Les souverainistes québécois, imbus qu'ils sont désormais de l'idéal républicain, négligent une chose: le regard que porte l'historien sur la nation est celui d'un homme appartenant à une nation forte, historiquement déterminée, sûre de son bon droit politique et qui, surtout, n'a plus à négocier ni à gérer la diversité des origines. Le pacte entre le peuple et l'Etat y est scellé depuis un bon moment. La France de Renan est une société fortement homogène quant à la culture et la langue, une société qui a réglé depuis un certain temps déjà les différends historiques qui ont pu dans le passé mettre un frein à l'uniformisation sociale. Au moment où écrit Renan, la France s'est constituée un espace public commun qui est de moins en moins remis en question. Et elle n'est pas seule: la plupart des autres pays européens ont, à l'époque, également réalisé leur intégration nationale. Il pouvait etre donc relativement facile et naturel de concevoir la nation comme le fit l'historien. D'autant plus qu'il est toujours plus simple d'en appeler à la solidarité des membres d'une communauté nationale (le vouloir vivre ensemble) lorsque l'ethnicité n'entre pas en jeu dans la dynamique des relations sociales. Les différends y seront économiques ou sociaux, mais ne porteront pour ainsi dire jamais sur le sens de la culture publique commune à laquelle la très grande majorité agrée sans sourciller. Jamais l'appartenance d'un membre de la communauté ne sera en litige du fait de sa différence ethnoculturelle; la question ne se pose même pas.

Le Québec n'est pas la France de Renan. Quoi qu'on en dise, la conjoncture actuelle ne réunit pas les conditions préalables à la postnationalisation de la subjectivité politique profonde des Québécois. La reconfiguration élargie, ouverte et transculturelle nécessaire à d'éventuels réaménagements du système politique ne va pas de soi.

17 Voir J. Saul, Voltaire's Bastards. The Dictatorship of Reason in the West, Toronto, Penguin Books, 1992. 
Le projet souverainiste stabilise la tension entre citoyenneté et nation en se réclamant d'une volonté de vie commune qui désigne la nation comme l'ensemble de tous ceux et celles qui résident et vivent sur le territoire du Québec, quelle que soit leur origine ethnoculturelle. Principe irréprochable s'il en est, mais il faut être prêt à endosser jusqu'au bout les conséquences d'une pareille reconceptualisation de la nation. Penser ainsi, c'est déjà définir non plus la nation, mais une sorte de communauté socio-politique neutre, presque désincarnée, désignée essentiellement à partir de mécanismes institutionnels ou politicojuridiques et administratifs précis. La primauté de la citoyenneté implique de la part des individus une allégeance à l'État qui surpasse toute autre allégeance. La citoyenneté libérale commande aux individus de faire de l'Etat leur base identitaire première, toute autre base identitaire (langue, ethnicité, culture, etc.) devenant accessoire ou complémentaire. Bref, la nation n'existe plus dans le projet souverainiste. Elle n'existe plus comme force sociale, comme moteur du mouvement de la société, comme justification de l'accomplissement collectif de la communauté imaginée d'origine - celle de la «nation naturelle», celle des francophones au profit de qui le mouvement nationaliste s'anime depuis plus de trois décennies. On demande à la nation de se ré-inventer, de se ré-imaginer non plus en fonction de critères identitaires intimes et personnels - c'est-à-dire articulés autour du vécu des individus dans leur rapport à un environnement culturel qui les marque historiquement --, mais bien en fonction de critères dépersonnalisés, enracinés dans une logique de mécanique institutionnelle.

C'est là le nœud du problème, qui témoigne du manque de réalisme politique des souverainistes québécois. Cette auto-réinvention de la communauté imaginée est hautement improbable au Québec, à tout le moins pas sur une base volontaire ou délibérée. Ce qui ne veut pas dire qu'elle soit totalement, irrémédiablement impossible. Il n'est pas interdit de l'envisager, mais alors seulement à très longue échéance, au prix du renoncement progressif aux composantes originelles de l'identité intime. Le jour que la souveraineté se sera accomplie sur les bases exclusives d'une rationalité purement étatique, la nation québécoise, telle que nous la connaissons aujourd'hui et à laquelle nous nous identifions d'emblée, aura disparu. Ce qui existera à la place, ce sera le «lieu Québec» au sein duquel des individus de diverses origines et de divers horizons auront finalement accepté de vivre ensemble une expérience sociale et politique par delà leurs différences identitaires. Ils se seront sans doute dotés d'une nouvelle identité, mais ce ne sera pas celle de la nation québécoise telle qu'elle est encore largement comprise aujourd'hui (ce «Nous-Autres» qui nous distingue et affirme 
notre unicité $\left.{ }^{18}\right)$. Ce sera une identité institutionnelle, essentiellement instrumentale, car c'est seulement sur ce terrain qu'il est possible d'opérationnaliser efficacement et en relative harmonie le pluralisme identitaire constitutif de la société québécoise. Dans pareil contexte, la «nation naturelle» n'aura plus d'influence ou elle n'aura d'influence que celle que la rationalité étatique lui aura accordée. En clair, cela veut dire que les Québécois francophones n'auront plus nécessairement le monopole de la nation québécoise - de la «québécitude». Le Québec ne sera plus, par définition, le lieu par excellence de la primauté de leur affirmation socioculturelle.

Il faut bien comprendre qu'il s'agit là de la conséquence obligée $\mathrm{du}$ projet théorico-politique des souverainistes québécois. D'aucuns pourront bien trouver que cette perspective n'a en soi rien de terrifiant ${ }^{19}$. Cependant, les conditions qui permettraient aux Québécois de vivre une telle situation et de l'accepter avec sérénité n'existent pas actuellement, pas plus qu'elles ne sont à la veille de poindre à l'horizon des possibles. La conjoncture générale rend impensable le passage de la nation-culture à la nation-raison auquel aspirent les souverainistes, et ce pour au moins deux raisons: la prédominance de la question identitaire dans la dynamique politique et le discours public contemporains; le raffermissement par l'État québécois de l'imaginaire socio-politique ethniciste.

\section{L'identitaire au cœur du politique}

La plupart des analyses récentes admettent d'ores et déjà la centralité de l'identitaire dans la dynamique politique des sociétés contemporaines ${ }^{20}$. Le Québec n'échappe pas à cette réalité. L'enchâssement dans la Constitution de la Charte canadienne des droits et libertés a convié l'ensemble des Canadiens à affirmer dans l'arène politique leurs

18 Voir P.-M. Lemaire, Nous Québécois, Montréal, Leméac, 1993.

19 Certains, en effet, la trouveraient plutôt encourageante. Un Lamberto Tassinari par exemple, directeur du magazine Vice Versa, se rangerait parmi eux, lui pour qui «résoudre le problème immigrant signifie se débarrasser de la "maladie Québec" et redéfinir de façon radicale l'identité québécoise». (L. Tassinari, «La ville continue. Montréal et l'expérience transculturelle de Vice Versa», Revue internationale d'action communautaire, vol. 21, no 61, 1989, p. 61).

20 Voir, par exemple, M. Freitag, «L'identité, l'altérité et le politique. Essai exploratoire de reconstruction conceptuelle-historique», Société, no 9, 1992, p. 1-55; A. O. Hirschman, «Social conflicts as pillars of democratic market society», Political Theory, vol. 22, no 2, 1994, p. 203-218; S. P. Huntington, «The clash of civilizations?», Foreign Affairs, vol. 72, no 3, p. 22-49; R. D. Kaplan, «The coming anarchy», The Atlantic Monthly, février 1994, p. 44-76; C. S. Maier, «Democracy and its discontents», Foreign Affairs, vol. 73, no 4, p. 48-64. 
identités particulières ${ }^{21}$. La Charte nous pousse en fait à vouloir l'avancement socio-économique de l'identité individuelle que nous nous reconnaissons et que nous voulons que les autres nous reconnaissent, quelles qu'en soient les composantes. Bien qu'elle n'ait pas encore toute l'autonomie et le poids légal de la Charte canadienne dans le contexte constitutionnel actuel, la Charte des droits et libertés de la personne du Québec ressortit à la même logique. Dans un Québec souverain où cette Charte servirait sans doute de base juridique et constitutionnelle, le sens général de la dynamique politique serait sensiblement le même que celui qui domine présentement au Canada.

Le fait est que nous évoluons dans un contexte politique qui glorifie notre singularité subjective. A priori, cela peut apparaître comme une avancée démocratique remarquable, mais ce n'est pas non plus sans poser quelques problèmes. Dans la course à l'affirmation identitaire, nous sommes en compétition, voire en confrontation, constante, les uns avec les autres pour la reconnaissance par l'État des impératifs et des besoins particuliers de notre identité, besoins dont nous réclamons la satisfaction en priorité. Au surplus, nous ne tolérons pas que s'instaure quelque forme que ce soit de hiérarchie des identités: en vertu du principe d'égalité formelle qui inspire nos chartes libérales, une identité vaut bien l'autre, et nous nous attendons à ce que l'État réponde également aux diverses identités qui le sollicitent. En fait, le processus politique au Canada et au Québec s'actualise désormais par des affrontements entre groupes et individus qui s'autodéfinissent et se mobilisent à l'identité (raciale, ethnique, linguistique, sexuelle, religieuse). Nous suivons en cela une tangente qu'empruntent aujourd'hui la plupart des démocraties libérales: il n'y a pas si longtemps, les conflits sociaux s'articulaient généralement autour de problématiques de nature économique, conflits que des compromis quantitatifs pouvaient en bout de piste résoudre; aujourd'hui, nos sociétés sont de plus en plus divisées selon des rivalités identitaires pour lesquelles il est difficile, voire impossible, de trouver un compromis. On peut négocier un contrat de travail ou une convention collective; on ne négocie pas sa singularité subjective ${ }^{22}$.

Le construit théorico-politique des souverainistes passe donc à côté de la réalité: il réclame la subsomption des identités singulières dans un

21 A. C. Cairns, «Citizens (outsiders) and governments (insiders) in Constitutionmaking: The case of Meech Lake», dans A. C. Cairns, Disruptions: Constitutional Struggles from the Charter to Meech Lake, textes colligés par D. E. Williams, Toronto, McClelland and Stewart, 1991, et "Constitutional minoritarianism in Canada», dans R. L. Watts et D. M. Brown (dir.), Canada: The State of the Federation 1990, Kingston, Institute of Intergovernmental Relations, 1990.

22 A. O. Hirschman, art. cité. 
grand tout universel et capable d'homogénéisation, alors même que la culture politique qui domine en ce moment tend désespérement vers l'inscription de l'hétérogène au centre du processus politique. Leur projet suppose un certain renoncement aux identités particulières que nous nous reconnaissons au profit de desseins socio-institutionnels posés d'emblée comme supérieurs parce qu'ils sont ancrés dans un quelconque universel rationnel. À moins d'un renversernent des pôles de la culture politique qui s'insinue dans la sphère publique depuis une dizaine d'années, il est difficile de concevoir comment ce renoncement peut s'accomplir.

Peut-on sérieusement imaginer les Québécois abandonner les termes d'une identité distincte pour la reconnaissance de laquelle ils luttent avec acharnement, mais sans succès satisfaisant, depuis plus de trente ans? Il n'est qu'à lire les mémoires présentés devant la Commission sur l'avenir politique et constitutionnel du Québec (commission BélangerCampeau) ou plus récemment devant les Commissions régionales sur l'avenir du Québec pour constater combien cela est improbable. Si les Franco-Québécois d'aujourd'hui n'adoptent plus l'attitude de repli sur soi et de refoulement identitaire qui caractérisait leurs pères, ils ne sont pas prêts pour autant à abandonner les attributs historiques de leur identité culturelle. La redéfinition de la nation ne doit pas entraîner la dissolution de la mémoire ancestrale et des acquis identitaires qui ont toujours distingué le groupe et qui continuent de le faire ${ }^{23}$. D'ailleurs, dans la plupart des cas, l'appui à la souveraineté est sensiblement motivé par les perspectives de protection linguistique et donc culturelle que l'option politique recèle ${ }^{24}$.

Dans le même ordre d'idée, on peut douter que les anglophones du Québec (autant les héritiers de Wolfe que les néo-Québécois) acceptent de bon gré d'intégrer un grand ensemble étatique, censément francophone, au détriment de leurs propres prétentions identitaires ${ }^{25}$. Il

23 J. Létourneau et J. Ruel, «Nous Autres les Québécois. Topiques du discours francoquébécois sur Soi et sur l'Autre dans les mémoires déposés devant la Commission Bélanger-Campeau», dans K. Fall, D. Simeoni et G. Vignaux (dir.), Mots représentations. Enjeux dans les contacts interethniques et interculturels, Ottawa, Presses de l'Université d'Ottawa, 1994, p. 283-307. Voir aussi J. Ruel, «Entre la rhétorique et la mémoire: usages du passé et références à l'histoire dans les mémoires déposés devant la Commission sur l'avenir politique et constitutionnel du Québec (1990)», Discours social/Social Discourse, vol. 6, no 1, 1994, p. 213-242.

24 R. Nadeau et C. J. Fleury, «Gains linguistiques anticipés et appui à la souveraineté du Québec», Revue canadienne de science politique, vol. 28, no 1, 1995, p. 35-50.

25 J. Legault, L'invention d'une minorité. Les Anglo-Québécois, Montréal, Boréal, 1992. 
en va de même pour les autochtones ${ }^{26}$. D'autant plus qu'en vertu des principes constitutionnels qui nous gouvernent ils ont toute la caution morale désirée pour refuser cette subsomption forcée (sans compter que pour ces groupes le seul grand ensemble étatique acceptable restera toujours le gouvernement fédéral).

Un espace symbolique commun est une condition minimale préalable à la mobilisation en vue d'un projet politique nationalitaire. À l'évidence, la grande diversité mémorielle des groupes ethnoculturels qui composent le Québec d'aujourd'hui témoigne de l'inexistence d'un tel espace symbolique. Les membres de chaque groupe agissent généralement à l'intérieur de sphères communicationnelles et culturelles qui leur sont propres, mais qui ne s'interpénètrent que fort peu et entre lesquelles les relations sont souvent larvées par le ressentiment et l'animosité 27 . L'absence d'un espace symbolique commun à l'intérieur duquel les divers groupes ethnoculturels du Québec se sentiraient en communauté communicationnelle ne peut que compliquer le procès de création d'une sphère étatique que tous accepteraient et dans laquelle tous se reconnaîtraient. Alors que la dynamique politique actuelle tend plutôt à repousser chacun dans ses derniers retranchements identitaires, cela augure mal pour l'instauration de la nation-raison.

\section{La gestion de l'altérité et la persistance de la nation-culture}

La centralité de l'identitaire au sein de la dynamique politique a ouvert la porte à l'émergence de l'hétérogène dans le paysage public québécois ${ }^{28}$ et oblige la prise en considération de l'altérité dans la recomposition de l'espace public. Depuis plusieurs années, l'État québécois s'est saisi de cette réalité en adoptant diverses politiques d'immigration et de gestion de la différence ${ }^{29}$ qui, de façon générale, témoignent d'intentions louables.

26 D. Salee, «Identities in conflict: The aboriginal question and the politics of recognition in Quebec», Ethnic and Racial Studies, vol. 18, no 2, 1995, p. 277-314; G. Alfred, Heeding the Voices of Our Ancestors, Toronto, Oxford University Press, 1995.

27 La réticence des jeunes allophones face à l'apprentissage et à l'usage du français (quand ce n'est pas le rejet pur et simple) en dit long à ce sujet. Voir F. Berger, «Les jeunes allophones boudent le français», La Presse, 12 août 1995, p. A1.

28 Voir D. Salée, «La mondialisation et la construction de l'identité au Québec», dans M. Elbaz, A. Fortin et G. Laforest (dir.), Identité et modernité: les leçons de l'expérience québécoise, Québec, Presses de l'Université Laval, à paraître.

29 Pour des analyses et des bilans de ces politiques, voir: D. Guay, Réflexions critiques sur les politiques ethniques du gouvernement fédéral et du gouvernement du Québec, note de recherche du CIDHICA, Montréal, CIDHICA, 1986; M. McAndrew, «Le traitement du 
Dans leur volonté de gommer le caractère ethnoculturel du nationalisme et de l'imaginaire étatique québécois, les souverainistes se méprennent souvent sur le rapport des Québécois francophones à l'altérité. Parce que les politiques étatiques sont officiellement empreintes d'ouverture à l'Autre et de tolérance, ils concluent volontiers à la «dé-ethnicisation» de l'imaginaire socio-politique québécois et considèrent la reconfiguration de la québécitude en dehors de l'horizon de la majorité francophone comme un fait accompli. Selon Jocelyn Létourneau et Jacinthe Ruel, un fossé important subsiste entre la conscience réelle du Sujet québécois et la situation idéale que dépeignent les souverainistes:

En fondant son rapport à l'altérité sur le rappel de ce qu'il est et de ce qu'il était (à travers ses traditions, sa langue et sa culture), [le Sujet québécois] établit dans son espace symbolique un filet sécuritaire qui le garde de toute errance du côté de l'hétérogénéité culturelle, errance implicitement envisagée comme une dérive incontrôlable vers un Ailleurs déstructurant au plan de l'identité ${ }^{30}$.

Il n'y a pas à s'en surprendre ni à s'en offusquer. C'est un peu dans l'ordre normal des choses si l'on considère les paramètres de développement social et étatique à l'intérieur desquels les Québécois évoluent depuis plus de trois décennies. En dépit du souci de rationalité étatique qui marque le Québec depuis la Révolution tranquille, en dépit de l'ouverture de l'État québécois du côté de la raison technocratique, il n'en demeure pas moins que celui-ci a été et continue d'être porteur de la communauté imaginée d'origine, porteur d'une conception ethnoculturelle précise de la québécitude. Au cours des trente dernières années, le Sujet québécois s'est servi de l'État provincial pour affirmer,

racisme, de l'immigration et de la réalité multi-ethnique dans les manuels scolaires francophones au Québec», Canadian Ethnic Studies/Études ethniques au Canada, vol. 18, no 2, 1986, p. 130-142; D. Juteau et M. McAndrew, «Projet national, immigration et intégration dans un Québec souverain», Sociologie et sociétés, vol. 24, no 2, 1992, p. 161-180; Groupe de recherche ethnicité et société, «Immigration et relations ethniques au Québec: un pluralisme en devenir», dans G. Daigle (dir.), ouvr. cité, p. 451-482; M. Labelle, «Immigration, intégration et identité au Québec», L'Action nationale, vol. 82, no 1,1992 , p. 39-53, et «Nation et ethnicité. Perspectives théoriques à propos du Québec», dans F.-R. Ouellette et C. Bariteau (dir.), Entre tradition et universalisme, Québec, IQRC, 1994, p. 37-74; D. Helly, «Politique québécoise face au "pluralisme culturel" et pistes de recherche: 1977-1990», dans J. W. Berry et J. A. Laponce (dir.), Ethnicity and Culture in Canada. The Research Landscape, Toronto, University of Toronto Press, 1994, p. 81-94.

30 J. Létourneau et J. Ruel, art. cité, p. 293-294 (les italiques sont de moi). Voir aussi J. Létourneau, «L'inscription de l'altérité au cœur du projet nationalitaire. Propos de conjoncture sur la situation québécoise», L'Homme et la société, vol. 26, no 103, 1992 , p. 57-65. 
confirmer et éventuellement imposer l'image nouvelle de lui-même qu'il voulait projeter ${ }^{31}$. L'État s'est fait le fer de lance de ses ambitions: par conséquent, État et ethnicité francophone au Québec sont intimement associés.

L'État québécois n'est pas post-national, pas plus qu'il n'est en train de le devenir. Certes, la rhétorique officielle actuelle, tout inclusive, toute rationnelle et toute dé-ethnicisée qu'elle prétend être, voudrait nous y faire croire. Dans les faits, bien que l'État ne fonctionne pas au profit exclusif des seuls francophones, il reste néanmoins établi selon des critères normatifs et institutionnels qui visent $a$ priori à satisfaire leurs aspirations particulières. En ce sens, si l'idée de souveraineté pour le Québec n'implique pas, par définition, une idéologie de l'exclusion culturelle, elle recouvre tout de même une certaine conception de la québécitude que partage plus naturellement les Québécois francophones. L'État québécois, souverain ou non, reste intimement lié à la qualité de Québécois francophone. C'est pour cette raison d'ailleurs que si peu d'anglophones et de membres des autres minorités culturelles s'y reconnaissent. Croire possible maintenant l'apparition d'une forme renouvelée de l'État québécois qui le situerait au-delà de la nation naturelle - dans «la suprématie du lien politico-juridique sur le lien culturel» - trahit une méconnaissance de l'histoire et du sens profond de l'imaginaire étatique québécois contemporain. C'est, par ailleurs, se méprendre sur la nature réelle des mesures et politiques étatiques de gestion de l'altérité.

Il est de bon ton au Québec de récuser la politique canadienne de multiculturalisme. Bien qu'elle soit axée sur l'égalité des cultures, les droits individuels et la célébration de la différence culturelle, on lui reproche généralement son association au bilinguisme officiel canadien et surtout sa tendance inhérente à ravaler le peuple québécois au rang de groupe ethnique parmi d'autres pour lequel aucun avantage politique ne saurait être consenti. L'idéologie du multiculturalisme canadien serait une «idéologie factice et pernicieuse qui a toujours favorisé l'anglicisation ${ }^{32}$. On lui préfère généralement une politique de convergence culturelle fondée sur l'apport enrichissant des cultures plurielles à la culture québécoise qui doit cependant rester essentiellement française et tenir lieu de pivot central de la culture publique commune. Au cours des dernières années, l'idée d'interculturalité est venue s'ajouter au principe de convergence culturelle pour proposer

31 J. Létourneau, «Québec d'après-guerre et mémoire collective de la technocratie», Cahiers internationaux de sociologie, vol. 90, 1991, p. 67-87, et «Le Québec moderne: un chapitre dans le grand récit collectif des Québécois», Revue française de science politique, vol. 42, no 5, 1992, p. 765-785.

32 P.-M. Lemaire, ouvr. cité, p. 125. 
«une démarche de compréhension dynamique des cultures d'origine des enfants migrants et éventuellement une éducation aux droits humains et à l'anti-racisme ${ }^{33}{ }^{\prime}$. L'approche québécoise face à l'altérité ethnoculturelle favorise l'intégration des minorités, dans le respect des droits de la personne, à un espace symbolique renouvelé - la nation renanienne - mais ancré dans l'expérience historique de la communauté imaginée d'origine. C'est, pour l'essentiel, la position des souverainistes.

En réalité, l’État québécois tient un discours éminemment ambigu à l'égard de l'altérité34. Du même souffle, il réclame la subsomption identitaire de l'Autre à la francité dominante et accueille avec une bienveillance de principe les manifestations de l'altérité sur la place publique.

Le slogan de la dernière campagne de publicité visuelle du gouvernement, "Le cœur québécois», est un exemple frappant de cette ambiguïté. Sur les panneaux et affiches qui composent le décor urbain de Montréal, on pouvait voir au cours des derniers mois des portraits de gens clairement (stéréo)typés aux traits raciaux et ethniques qui marquent de manière non équivoque leur différence par rapport à la majorité d'origine européenne de la société québécoise. Sur chacun de ces panneaux, on pouvait lire une mention qui variait selon les caractéristiques raciales ou culturelles du sujet présenté. Ainsi: «Les yeux en amande... le cœur québécois» (dans le cas d'une jeune femme asiatique); «Les cheveux bouclés... le cœur québécois» (dans le cas d'un enfant de race noire); et ainsi de suite.

Cette publicité procède sans doute d'une bonne intention. L'État cherche à favoriser l'intégration des immigrants au sein de la société québécoise d'une manière qui se veut non agressive et respectueuse. On signifie à l'Autre qu'en dépit de sa différence ethnoculturelle et raciale évidente on est prêt à l'accueillir chaleureusement, comme s'il faisait déjà partie de la famille - «Le cœur québécois». D'ailleurs, parmi la série de portraits que comprend la campagne publicitaire, on trouve

33 M. Labelle, «Immigration, intégration...», art. cité, p. 51.

34 Il n'est pas dans mon intention d'épiloguer ici sur les mérites et désavantages des approches canadienne et québécoise en matière de gestion de l'altérité ethnoculturelle. Les propos critiques tenus ici à l'égard de l'approche québécoise ne sauraient être interprétés comme une adhésion au multiculturalisme canadien. Dès que l'on fait abstraction des considérations politiques - voire politiciennes - qui motivent l'une et l'autre approche, on se retrouve en bout de piste devant deux visions qui ne cherchent que l'intégration-uniformisation de l'Autre dans le cadre d'une sphère publique qui, en définitive, reste définie par la majorité historique. Au chapitre des résultats politiques concrets auxquels elles aspirent, les deux approches s'équivalent. 
celui d'une jeune femme de race blanche (sous-entendre québécoise francophone), tout sourire et bras ouverts. Dans la mesure où la publicité interpelle l'Autre, elle a pour objectif clair d'élargir, au moins théoriquement, l'espace public et l'expression de la citoyenneté, d'instituer une authentique démarche d'inclusion sociétale. La publicité s'adresse aussi aux Québécois francophones: elle leur montre que le Québec, ce n'est plus seulement les «Canadiens français»; elle cherche à faire prendre conscience du caractère positif de l'altérité (tous les visages représentés sont fort sympathiques) et à bien faire comprendre que l'Autre est aussi québécois. Bref, une publicité en apparence irréprochable, soucieuse d'afficher le caractère pluraliste, démocratique et ouvert de la société québécoise.

Pourtant, le message, qui dérive de la philosophie étatique de gestion de l'altérité, n'est pas aussi innocent qu'il semble et peut se prêter à une tout autre interprétation. Au-delà du contexte d'harmonie interculturelle qu'il prétend créer, il intime l'Autre à se faire québécois, à voir, à sentir les choses avec «un cœur québécois». Sur le ton de la cordialité, on prend fait et acte de la différence du porteur d'altérité, en l'invitant toutefois à se fondre au plus tôt dans le tout social québécois; un tout social préétabli, prédéterminé sur lequel l'Autre a finalement bien peu d'influence. Sous le couvert du respect de la différence, l'État québécois impose en fait un ordre culturel fondé sur une philosophie du «Même». Il cherche à fabriquer des similitudes, des corrélations et des rapports consensuels de manière à ré-identifier la nation sans perdre le contrôle sur le contenu, c'est-à-dire sans que ce contenu prenne une forme que la communauté imaginée d'origine aurait peine à reconnaître. L'État joue donc de prudence sur deux plans à la fois: il conforte la majorité dans sa conviction qu'elle doit tenir un rôle central dans la construction de l'espace public et y occuper une place privilégiée; il cherche à convaincre l'Autre que sa contribution est néanmoins importante et qu'il ne saurait être question de l'exclure démocratie oblige - à condition qu'il consente au procès de subsomption identitaire auquel il est convié.

Cette démarche n'est pas sans limiter les possibilités d'actualisation du passage souhaité de la nation-culture à la nation-raison. D'abord, elle est susceptible d'entretenir un sentiment de perplexité au sein des minorités culturelles. Pourquoi le porteur d'altérité devrait-il se fondre dans un grand tout social au détriment éventuel de sa différence alors même que le discours officiel accepte, conforte, voire protège cette dite différence? Ou encore pourquoi, si sa différence doit être source d'enrichissement pour la société québécoise, n'est-il pas plus directement partie prenante du procès de redéfinition de la nation? Pourquoi devrait-il simplement endosser aveuglément le projet social des élites québécoises qui auraient plutôt tendance à le tenir à l'écart 
des débats autour de la recomposition de l'espace public? Ces questions se posent d'autant plus que la culture politique ambiante l'encouragerait plutôt à résister aux grands projets universalistes et que certains groupes ethnoculturels jouissent d'une situation de complétude institutionnelle qui leur donne les outils de cette résistance ${ }^{35}$. En d'autres mots, tant que la convergence culturelle continuera d'apparaître au porteur d'altérité comme une forme déguisée de tentative d'assimilation, les bénéfices qu'il pourrait retirer du grand projet de la nation-raison seront loin de s'imposer d'évidence.

En fait, l'Autre est invité à contribuer à la société québécoise, mais selon les termes d'un espace symbolique et communicationnel qui lui échappent. En bout de piste, il se voit forcé de se retrancher dans un espace qui lui est propre, mais qui le renvoie aux référents ethnoculturels de son altérité et le maintient à la périphérie de l'espace dominant. Eût-il voulu effacer ces référents de sa mémoire qu'ils lui seraient ré-imposés par la force des circonstances de la dynamique politique ${ }^{36}$. La campagne publicitaire «Le cœur québécois» rappelle à l'Autre sa différence et son altérité et crée les conditions d'un débat social qui ne peut que s'articuler en des termes ethnoculturalistes. En ce sens, les politiques étatiques de gestion de l'altérité consolident, au sein tant de la majorité que des minorités culturelles, le procès d'autoperception et orientent le regard que l'on porte sur les autres d'une façon qui interpelle nécessairement l'ethnicité et la culture. Loin d'atténuer la différenciation ethnoculturaliste nécessaire à l'accomplissement de la nation-raison, les politiques étatiques actuelles contribuent au développement d'une conscience ethnoculturelle au sein des diverses composantes de la population.

Dans sa gestion de l'altérité l'État québécois reste donc ethniciste; il impose un cadre de références ethnicistes au discours public et force tout un chacun à se positionner à l'intérieur de ce cadre. Malgré la volonté de passer à la nation-raison, l'imaginaire étatique reste accroché à l'État-culture. Porteurs d'une identité que le contexte politique canadien s'est toujours ingénié à banaliser, les Québécois sont encore

35 Voir M. Labelle, G. Beaudet, J. Lévy et F. Tardif, «La question nationale dans le discours de leaders d'associations ethniques de la région de Montréal», Cahiers de recherche sociologique, no 20, 1993, p. 85-111; M. Labelle, M. Therrien et J. Lévy, «Le discours des leaders d'associations ethniques de la région de Montréal», Revue européenne des migrations internationales, vol. 10, no 2, 1994, p. 119-146.

36 Faut-il s'étonner dès lors de ce qu'un sondage du ministère des Communautés culturelles et de l'Immigration mené auprès de minorités culturelles révélait il y a quelques années que trois personnes sur quatre au sein de ces communautés s'identifiaient plus volontiers à leur ethnie d'origine qu'à la québécitude? (P. Cauchon, «Le brouillage des identités», Le Devoir, 7 octobre 1992, p. B1.) 
incapables de se placer dans un cadre conceptuel qui fasse abstraction de la culture et de l'ethnicité. À vrai dire, les conditions objectives de leur développement historique propre ne leur permettent pas encore de transcender leur spécificité ethnique dans la recomposition de l'espace public. Admettre la chose, ce n'est pas taxer les Québécois d'ethnocentrisme, c'est tout simplement prendre fait et acte d'une réalité plutôt que de la nier. L'ethnicité et, de façon plus générale, les particularismes identitaires ne disparaîtront pas comme facteurs pertinents de la dynamique politique simplement parce qu'on le souhaite. Il faut plutôt apprendre à composer avec ses aboutissants.

\section{La peur de l'ethnicité et la fiction rationaliste}

À la lumière des atrocités et des excès commis un peu partout au nom de l'ethnicité ou de la défense de spécificités culturelles, on comprendra aisément que les souverainistes cherchent aujourd'hui à désamorcer le contenu traditionnellement culturaliste du nationalisme québécois et à ramener le débat autour de la question de la nation québécoise sur le terrain plus neutre et prétendument plus objectif de la raison. Celui-ci offre en effet l'illusion confortante de possibles consensus et de réconciliations qui mettraient fin à la dynamique fragmentaire qu'impose la politisation des particularismes.

En choisissant ce terrain, les souverainistes s'inscrivent carrément dans la mouvance de la pensée et des pratiques du libéralisme contemporain. Terrain miné s'il en est, dont la topographie est marquée au coin de la fiction égalitariste et du leurre de l'unanimisme démocratique. Miné, parce qu'il participe d'une conception mystifiante du social et des rapports sociaux. La pensée libérale, en effet, évacue le politique: elle nie que les relations sociales sont des relations de pouvoir, et donc d'exclusion, et préfère ignorer le caractère éminemment public des antagonismes sociaux, les reléguant plutôt à la sphère privée. Rien ne saurait être plus chimérique que cette vision des choses. Toute forme d'antagonisme social (ethnique, religieux, économique, moral, éthique) se transmue en antagonisme politique dès lors que la vision libérale mobilise suffisamment d'énergie et de ressources humaines pour s'inscrire dans l'arène publique. Aussi l'idéal souverainiste de nationraison, modelée sur un imaginaire étatique rationaliste, repose-t-il sur le mirage d'un consensualisme mythique.

Le libéralisme contemporain et l'imaginaire étatique qu'il nourrit sont piégés. De tout temps, le libéralisme s'est élaboré dans la tension entre la liberté de l'individu et l'intégrité du corps étatique. Cette tension pénètre et module la psyché politique occidentale et marque le concept de souveraineté d'ambiguités jamais résolues. L'idée d'un État 
fort, capable d'homogénéisation et centralisé s'est imposée dès le début au concept de souveraineté et a dominé jusqu'au XXe siècle en partie en raison des progrès réalisés par l'État-nation. La souveraineté nationale est devenue un principe structurateur d'organisation politique, et l'État souverain, le véhicule principal de gestion et même de résolution des conflits de classes, ethniques ou religieux au sein des nations. L'Étatnation souverain a pris forme à partir du consensus qui s'est forgé autour de l'impérieuse nécessité de constituer des communautés politiques unifiées afin de rencontrer les exigences du marché. Mais, en arrière-plan, n'a jamais cessé de se profiler et de se développer l'idée de souveraineté en référence directe à la liberté et à l'autonomie de l'individu. Pour plusieurs, malgré la force institutionnelle de l'État, c'est l'individu et non l'État qui est souverain. La contrainte réelle qui s'impose au citoyen n'est pas l'obligation légale d'obéir au gouvernement, mais l'obligation morale de poursuivre ce qui lui semble constituer l'idéal de la justice ${ }^{37}$.

Du fait de son ambiguïté originelle constitutive, on pourrait presque dire que, au moment même de son apparition dans la pensée politique occidentale, la notion de souveraineté était pratiquement vouée à la remise en question éventuelle de sa légitimité, non pas tant comme concept structurant du politique, mais surtout dans son contenu si intimement lié à l'État universel, unitaire, unifié et centralisé.

La communauté politique est généralement traversée de conflits qui débouchent inévitablement sur la fragmentation du pouvoir, c'est-à-dire qui remettent constamment en question la place des détenteurs du pouvoir socio-économique et les organisations socio-politiques qu'ils dirigent. Ces conflits prennent leur source dans la volonté des individus et des groupes écartés du pouvoir de s'aménager des sphères d'autonomie et d'action qui ne doivent rien aux institutions dominantes, ou encore dans leur désir de remanier ces institutions selon leurs aspirations propres. Ces mêmes conflits mettent donc en jeu la souveraineté en ce qu'elle symbolise un corpus politique unitaire, unifié et centralisé. Ils consacrent en quelque sorte le divorce entre la souveraineté étatique et la souveraineté populaire et les notions concomitantes de pluralisme, d'autonomie, de liberté et d'imputabilité. C'est bien pour cela que les discours souverainistes, dans la mesure où ils prennent ancrage dans l'État, reposent essentiellement sur un appel au consensus national. Il s'agit d'amenuiser, voire de désamorcer, le plus possible les velléités de conflits sociétaux qui menacent l'atteinte

37 J. A. Camilleri, «Rethinking sovereignty in a shrinking, fragmented world», dans R. B. J. Walker et S. H. Mendlovitz (dir.), Contending Sovereignties. Redefining Political Community, Boulder, Lynne Rienner Publishers, 1990, p. 13-44. 
des objectifs d'universalisation et d'aplanissement que contient nécessairement tout projet de souveraineté étatique.

Toutefois, les sociétés contemporaines sont arrivées au terme de l'impérialisme de l'universel et de l'homogène. À partir de la Seconde Guerre mondiale en particulier, les sociétés occidentales ont assisté à l'élargissement constant et rapide des pratiques démocratiques et d'inclusion sociétale. D'aucuns diront que l'on est encore loin de la perfection à cet égard, et c'est vrai. Cependant, il faut tout de même admettre que la généralisation du droit de vote, le développement de fonctions providentialistes au sein de l'État moderne, les chartes de droits et de libertés, la multiplication de politiques et de mesures reflétant un plus grand souci de justice sociale ont sensiblement réduit l'écart socio-politique entre dominants et dominés, entre majorités et minorités, entre nantis et démunis, entre l'élite et la masse.

C'est là où l'affaire se complique. Non pas que la réduction de l'écart fût une erreur, mais bien parce que l'élargissement de la sphère démocratique a nécessairement créé des attentes. Si l'État dit à la femme sous-payée ou sous-employée, au Noir ghettoïsé, à l'autochtone dépossédé et acculturé ou à toute autre victime de l'inégalité socioéconomique inhérente à la société de marché que justifie le paradigme libéral: «Â partir de maintenant, vous avez des droits véritables, le droit à la dignité, le droit à un traitement équitable, à la reconnaissance de votre égalité devant la loi, à un emploi rémunéré selon des critères d'équité et d'égalité; mieux, vous avez le droit à votre différence ethnoculturelle, religieuse, linguistique, vous avez le droit d'exister comme communauté nationale, nous reconnaissons cette différence et nous vous encourageons à l'exprimer fièrement», il faut bien s'attendre à ce que ces droits étalés à la face des minorités, des marginaux, des exclus et des opprimés soient pour eux plus que de simples affirmations. Les affirmations ne suffisent plus. Les déclassés d'hier entendent bien maintenant bénéficier pleinement de ces droits qu'on leur reconnaît et n'hésitent pas à recourir à la rhétorique de la démocratie et de l'égalitarisme pour que l'État libéral leur accorde des espaces d'autonomie qu'il n'avait pas vraiment envisagé de consentir.

C'est là où le bât blesse. La société et l'État libéraux sont incapables d'aller jusqu'au bout de la logique individualisante sans risquer leur propre déconstruction. On dira à l'autochtone qu'il est membre d'une nation que l'on reconnaît, mais on ne lui abandonnera aucun territoire sur lequel exercer sa pleine souveraineté politique, culturelle ou administrative; on dira à l'immigrant qu'il est citoyen à part entière à condition qu'il s'intègre ou s'assimile; on reconnaîtra à la femme la pleine égalité, à condition que cela ne remette pas trop en question le 
modèle patriarcal d'exercice du pouvoir et d'autorité sur lequel s'appuie l'État.

Les attentes qu'a engendrées le paradigme libéral-démocratique au cours des dernières décennies sont énormes. Les groupes et mouvements sociaux réclament un espace identitaire qui s'inscrive en dehors de la communauté politique telle qu'elle s'est constituée historiquement, car celle-ci correspond ou satisfait de moins en moins aux impératifs et priorités des corpus identitaires en émergence. C'est là le grand impensé du paradigme libéral. Trop imbu de sa vision universaliste et homogénéisante de la communauté politique et, concomitamment, de la souveraineté, l'État libéral n'a pas prévu de sphères d'autonomie et de différence. Au mieux, il se cantonne derrière une rhétorique séduisante de reconnaissance de la différence, qui n'est cependant que rarement appuyée par des mesures concrètes à la hauteur des espoirs suscités par cette même rhétorique. Les libéraux ne comprennent pas que désormais l'on n'aspire plus nécessairement qu'à la souveraineté (étatique) mais bien à des souverainetés.

Dans ce contexte général, la redéfinition rationaliste de la nation québécoise est donc piégée aussi et se confine dans les limites de l'impensé libéral. En prétendant à un universel rationnel, les souverainistes, bien qu'ils s'en défendent, gomment en réalité les différences constitutives de la société québécoise et avouent implicitement participer en cela à la grande entreprise libérale de mystification politique. Quel que soit l'angle sous lequel on l'aborde, l'établissement de la nation-raison est un acte de pouvoir enraciné dans la volonté d'hégémonie sociale de qui l'articule. Un acte de pouvoir qui s'inscrit à contre-courant de la dynamique politique actuelle et qui reflète essentiellement une hantise à peine voilée de la fragmentation sociale. Or le Québec est maintenant composé de fragments identitaires disparates qui, dans l'atmosphère déconstructiviste de notre époque, ne peuvent - ni ne veulent, bien souvent - se recomposer en une totalité nationale au sens de la nation-raison. Cela ne changera pas. Le projet de reconfiguration nationale que formulent aujourd'hui les souverainistes ne peut donc se réaliser à terme que dans l'oblitération effective de l'altérité. Étant donné la force des prétentions identitaires qui marquent la dynamique politique contemporaine, tenter de recomposer l'espace public en fonction de paramètres unitaristes et d'une philosophie du «Même» ne peut se faire qu'à coups de coercition et de subordination dirigés vers les identités en émergence. Il n'y a pas d'autres issues à l'intérieur de la logique opérationnelle ensembliste de l'universel rationnel. Pareille démarche s'inscrit forcément à l'opposé d'une véritable démocratie et d'une citoyenneté élargie. 


\section{Quel espace public?}

La peur de l'ethnicité n'est pas sans participer d'une certaine hypocrisie intellectuelle. La décomposition et le démembrement de l'unité politique collective transcendantale (l'État) auxquels, croit-on, les manifestations de l'ethnicité pourraient conduire sont, en fait, inscrits en creux dans la logique de développement socio-politique des sociétés libérales modernes. La pensée libérale démocratique a réussi à convaincre l'Occident qu'elle constitue le stade ultime d'accomplissement sociétal, ce qui a conduit nos sociétés à ce point de rupture. La célébration de l'identité singulière, à laquelle les procès de fragmentation et de politisation des particularismes en cours sont souvent attribués, n'est que la résultante logique de la philosophie individualiste du libéralisme contemporain. Il ne s'agit pas simplement d'une aberration temporaire ni d'une dérive momentanée. Elle est installée à demeure. Repousser, nier ou chercher à désamorcer le phénomène ne servirait à rien, sinon que d'en exacerber les manifestations. Chaque agent social est doté d'une identité propre qui le marque et détermine les modalités de son insertion dans l'espace public. Le forcer à oublier cette identité au profit d'une identité autre, censément supérieure ou désincarnée, lui paraîtra inévitablement rébarbatif et contre-nature.

Il s'agit donc de composer avec la dynamique identitaire, de la percevoir comme une réalité irréfragable, avec tout ce qu'elle peut receler de potentiel antagonique. Ainsi, dans un espace public véritablement démocratique, il ne s'agit pas de masquer l'ethnicité ou tout autre particularisme identitaire, mais bien d'accepter son existence comme composante en même temps inhérente à cet espace et constitutive de celui-ci. Les particularismes n'ont en soi rien de menaçant; ils ne le deviennent que dans la mesure où on veut bien croire qu'ils le sont.

Repositionner ainsi l'ethnicité et les particularismes identitaires suppose inévitablement la construction d'un imaginaire politique autre que celui qui domine actuellement les sociétés libérales. Cela implique qu'il faille procéder à la déconstruction du corpus institutionnel et de l'ethos politique qui les marquent. En clair, il faut réintroduire le politique dans nos rapports sociaux, admettre qu'il est «une dimension inhérente à toute société humaine et qui relève de notre condition ontologique elle-même ${ }^{38}$ »; il faut reconnaître que tout consensus est fondé sur l'imposition d'une volonté hégémonique et sur des actes d'exclusion, et donc refuser les consensus rationnels; il faut concevoir

38 C. Mouffe, «Pour un pluralisme agonistique», Revue du Mauss semestrielle, no 2, 1993, p. 101. 
l'identité comme un rapport de forces inscrit au cœur du politique et consentir à ce que l'hybridité, le multiple et un polymorphisme accru du social s'imposent comme les conditions fondamentales d'une véritable démarche démocratique et d'une vision élargie de la citoyenneté. En termes politiques concrets, cela suppose une configuration institutionnelle flexible qui ne banalise pas les différences et l'altérité, qui ne hiérarchise pas les identités et qui ne soit pas fondée sur une conception prétablie de l'espace public ${ }^{39}$.

C'est là le véritable enjeu lié à la recomposition de l'espace public québécois, souveraineté ou pas. Chercher à nier l'ethnicité comme le propose le projet théorico-politique des souverainistes, c'est fermer les yeux sur les fondements de la réalité politique du Québec; c'est se mentir à soi-même. Pire, c'est faire fausse route. L'espace public à construire au Québec doit s'organiser selon une grammaire politique aux accents nouveaux. Minimalement, cela implique l'abandon de la «Nation» comme pivot axiomatique de la communauté politique et la reconnaissance non équivoque de la société québécoise comme société multinationale ${ }^{40}$. De façon plus générale, cela signifie travailler à l'élargissement du débat social vers une remise en question fondamentale, voire un rejet, des paramètres du libéralisme contemporain.

Il revient aux forces sociales revendiquant le changement et le progrès social d'engager la reconfiguration de l'espace public sur des bases nouvelles et de formuler un discours neuf qui ne s'embarrasse pas du libéralisme faussement pluraliste dont le souverainisme actuel s'alimente. Sinon, le Québec se condamne à refaire le même parcours limité et limitatif des Etats-nations modernes dont le modèle est arrivé au terme de son temps utile. Ironiquement, il ne fera qu'approfondir le sillon déjà creusé par l'État canadien, celui-là même contre lequel, pourtant, toute la démarche émancipatoire québécoise des trente dernières années s'est activée. En fait, eu égard à sa propre expérience d'exclusion et de non-reconnaissance de sa différence au sein de l'État canadien, le Québec devrait précisément tendre aujourd'hui vers la recherche de formules politiques qui rompent avec l'illusion démocratique de l'universel rationnel. C'est dans cette direction que devrait plutôt se tourner le «vrai changement».

Daniel SALÉE

École des affaires publiques et communautaires Université Concordia

\footnotetext{
39 W. E. Connolly, Identity/Difference. Democratic Negotiations of Political Paradox, Ithaca, Cornell University Press, 1991.

$40 \mathrm{Je}$ rejoins en cela mon collègue Guy Laforest. Voir son «Identité et pluralisme libéral au Québec», dans S. Langlois (dir.), Identités et cultures nationales, Québec, Presses de l'Université Laval, 1995, p. 324-325.
} 


\section{2 Être ou ne pas être québécois}

\section{Résumé}

Ce texte propose une lecture critique du discours souverainiste sur la recomposition de l'espace public national québécois. L'auteur soutient essentiellement que la redéfinition de la nation souhaitée par les souverainistes québécois selon laquelle le Québec doit tendre vers l'accomplissement d'une nation dé-ethnicisée, fondée sur une logique civique et rationnelle, est en porte-à-faux par rapport à l'imaginaire social des Québécois et la réalité politique du Québec. Sous couvert d'adhésion à la raison universelle, cette redéfinition repose sur une mise au ban de l'ethnicité et des particularismes culturels et participe d'une volonté de subsomption à la fois de l'identité historique des Québécois et des identités autres qui composent désormais le tissu social du Québec en un espace public post-national et homogénéisant. Contrairement aux apparences, le sens de la nation qui dérive du projet théorico-politique des souverainistes cache en réalité un acte d'exclusion et des prétentions hégémoniques au profit de la nation historique. L'auteur soutient qu'il ne sert à rien d'évacuer la différence et l'altérité inhérentes à la dynamique politique québécoise. L'ethnicité et la culture sont des faits de conscience incontournables. Un véritable projet d'avant-garde de recomposition de l'espace public au Québec devrait plutôt chercher à en accepter les aboutissants et à réaliser une configuration institutionnelle flexible qui ne banalise pas l'altérité et la différence, qui ne hiérarchise pas les identités et qui ne soit pas fondée sur une conception préétablie de l'espace public. Cela implique l'abandon de la «Nation» cornme pivot axiomatique de la communauté politique et une remise en question fondamentale des paramètres du libéralisme contemporain.

Mots-clés: Québec, souveraineté, nation, ethnicité, culture, identité, altérité, espace public, libéralisme, État.

\section{Summary}

This article proposes a critical reading of pro-sovereignty discourse on the recomposition of Quebecois national public space. Essentially, it maintains that the redefinition of the nation desired by advocates of Quebec sovereignty, which pushes Quebec to the realization of a deethnicized nation, predicated on a civic, rational logic, is out of step with the Quebecois social imaginary and the political reality of Quebec. Cloaked in an adherence to universal reason, this redefinition essentially banishes ethnicity and cultural particularisms and advocates subsuming both the historical identity of the Quebecois and other identities now constitutive of Quebec's social fabric by an homogenizing, postnational public space. Contrary to appearances, the sense of the nation emanating from the pro-sovereignty theoretico-political project actually 